\title{
GENERALIZED HILBERT INTEGRAL OPERATORS ON THE HERZ SPACES
}

\author{
KUANG JICHANG
}

\begin{abstract}
This paper gives some necessary and sufficient conditions for the generalized Hilbert integral operators to be bounded on the Herz spaces. The corresponding new operator norm inequalities are obtained.
\end{abstract}

\section{Introduction}

Considerable attention has been given to the classical Hilbert operator $T$ defined by

$$
T(f, x)=\int_{0}^{\infty}\left(\frac{1}{x+y}\right) f(y) d y
$$

and the classical Hilbert inequality

$$
\|T f\|_{p} \leq \frac{\pi}{\sin (\pi / p)}\|f\|_{p}, \text { for } 1<p<\infty
$$

where $\|f\|_{p}=\left(\int_{0}^{\infty}|f(x)|^{p} d x\right)^{1 / p}$ and the constant factors $\pi / \sin (\pi / p)$ is the best value (see [1]). In view of the mathematical importance and applications, considerable attention has also been given to various improvements, refinements and extensions of many inequalities by various authors (see e.g., [3, 4, 5, 6, 7, 8, 9, 10, 11] and the references cited therein).However, hardly any work was done on inequalities on the Herz spaces. It is well-known that the Herz spaces play an important role in characterizing the properties of functions and multipliers on the classical Hardy spaces. In recent years, a series of papers have paid more attention to the study of the Herz spaces themselves (see [12, 13]). The aim of this paper is to establish some new inequalities related to the generalized Hilbert integral operator

$$
T(f, x)=\int_{0}^{\infty} K(x, y) f(y) d y
$$

with the general kernel $K(x, y)$. We obtain some necessary and sufficient conditions for the generalized Hilbert integral operator $T$ to be bounded on the Herz spaces. The corresponding new operator norm inequalities are obtained.

Received April 30, 2008.

2000 Mathematics Subject Classification. 26D10, 47A30.

Key words and phrases. Generalized Hilbert integral operator, Herz spaces, norm. 


\section{Definitions and statement of the main results}

Definition 1. Let $\alpha \in R, 0<p, q<\infty, B_{k}=\left\{x \in R^{n}:|x| \leq 2^{k}\right\}, D_{k}=B_{k}-B_{k-1}$ $(k \in Z), \varphi_{k}=\varphi_{D_{k}}$ denote the characteristic function of the set $D_{k}$.

(1) The homogeneous Herz space $\dot{K}_{q}^{\alpha, p}\left(R^{n}\right)$ is defined by [12]:

$$
\dot{K}_{q}^{\alpha, p}\left(R^{n}\right)=\left\{f \in L_{l o c}^{q}\left(R^{n}-\{0\}\right):\|f\|_{\dot{k}_{q}^{\alpha, p}}\left(R^{n}\right)<\infty\right\},
$$

where

$$
\|f\|_{\dot{K}_{q}^{\alpha, p}\left(R^{n}\right)}=\left\{\sum_{k=-\infty}^{\infty} 2^{k \alpha p}\left\|f \varphi_{k}\right\|_{q}^{p}\right\}^{1 / p}
$$

(2) The homogeneous Herz type space $\dot{K}_{q}^{\alpha, p}(\omega)$ is defined by

$$
\dot{K}_{q}^{\alpha, p}(\omega)=\left\{f \in L_{l o c}^{q}\left(R^{n}-\{0\}\right):\|f\|_{\dot{K}_{q}^{\alpha, p}(\omega)}<\infty\right\},
$$

where

$$
\|f\|_{\dot{K}_{q}^{\alpha, p}(\omega)}=\left\{\sum_{k=-\infty}^{\infty} 2^{k \alpha p}\left\|f \varphi_{k}\right\|_{q, \omega}^{p}\right\}^{1 / p}
$$

We can similarly define the non-homogeneous Herz space $K_{q}^{\alpha, p}\left(R^{n}\right)$ and $K_{q}^{\alpha, p}(\omega)$. It is easy to see that when $p=q$, we have $\dot{K}_{p}^{0, p}\left(R^{n}\right)=K_{p}^{0, p}\left(R^{n}\right)=L^{p}\left(R^{n}\right)$, and $\dot{K}_{p}^{\alpha / p, p}\left(R^{n}\right)=K_{p}^{\alpha / p, p}\left(R^{n}\right)=L^{p}\left(|x|^{\alpha} d x\right)$. Throughout this paper, we write

$$
\|f\|_{p, \omega}=\left(\int_{R^{n}}|f(x)|^{p} \omega(x) d x\right)^{1 / p} .
$$

Our main results are the following two theorems:

Theorem 1. Let $\alpha \in R, 0<p<\infty, \lambda>0$ and $1 \leq q<\infty, \omega(x)=x^{(1-\lambda) q}$. Let $F(p)=\int_{0}^{\infty} t^{(\lambda-1-1 / q) p} K^{p}(1, t) d t$ and $K(x, y)$ be a nonnegative measurable function on $(0, \infty) \times(0, \infty)$ which satisfies the following conditions:

(1) $K(t x, t y)=t^{-\lambda} K(x, y)$ for all $t>0$;

(2) There exist the constants $C_{1}(p), C_{2}(p)>0$, such that

$$
\begin{aligned}
& F(1) \leq C_{1}(p)\{F(p)\}^{1 / p}, \quad \text { for } 1 \leq p<\infty ; \\
& F(1) \leq C_{2}(p)\{F(p)\}^{1 / p}, \quad \text { for } 0<p<1 .
\end{aligned}
$$

Then the generalized Hilbert integral operator $T$ defined by (1.3) is a bounded operator from $\dot{K}_{q}^{\alpha, p}(\omega)$ into $\dot{K}_{q}^{\alpha, p}\left(R_{+}^{1}\right)$ if and only if

$$
\int_{0}^{\infty} t^{\lambda-\alpha-1-(1 / q)} K(1, t) d t<\infty .
$$


Moreover, when (2.7) holds, the operator norm $\|T\|$ of $T$ on $\dot{K}_{q}^{\alpha, p}(\omega)$ satisfies the following inequality:

$$
\int_{0}^{\infty} t^{\lambda-\alpha-1-(1 / q)} K(1, t) d t \leq\|T\| \leq C(p, \alpha) \int_{0}^{\infty} t^{\lambda-\alpha-1-(1 / q)} K(1, t) d t,
$$

where

$$
C(p, \alpha)=\left\{\begin{array}{ll}
2^{1 / p-1}\left\{C_{1}(1 / p)\right\}^{1 / p} C_{2}(p)\left(1+2^{|\alpha|}\right), & 0<p<1 \\
2^{1-1 / p} C_{1}(p)\left\{C_{2}(1 / p)\right\}^{1 / p}\left(1+2^{|\alpha|}\right), & 1 \leq p<\infty
\end{array} .\right.
$$

Corollary 2. If $t^{\lambda-1-1 / q} K(1, t)$ is concave function on $(0, \infty)$ and $K(1, t)$ has compact support: supp $K(1, t) \subset[0, b]$, then by (2.5), (2.6), Hölder inequality and Theorem 24 in $\left[2, \S 43\right.$, pp.43-44], we obtain $C_{1}(p)=b^{1-1 / p}$ and $C_{2}(p)=2^{-1}(1+p)^{1 / p} b^{1-1 / p}$, thus by (2.9), we get

$$
C(p, \alpha)= \begin{cases}2^{1 / p-2}(1+p)^{1 / p}\left(1+2^{|\alpha|}\right), & 0<p<1 \\ 2^{1-2 / p}(1+1 / p)\left(1+2^{|\alpha|}\right), & 1 \leq p<\infty\end{cases}
$$

Theorem 3. Let $\alpha \in R, 1 \leq p<\infty, \lambda>0$ and $K(x, y)$ be a nonnegative measurable function defined on $(0, \infty) \times(0, \infty)$. Which satisfies the following condition: $K(t x, t y)=$ $t^{-\lambda} K(x, y)$ for all $t>0$. Let $\omega_{1}(x)=x^{\alpha}$ and $\omega_{2}(x)=x^{(1-\lambda) p+\alpha}$. Then the generalized Hilbert integral operator $T$ is defined by (1.3) is a bounded operator from $L^{p}\left(\omega_{2}\right)$ into $L^{p}\left(\omega_{1}\right)$ if and only if

$$
\int_{0}^{\infty} t^{\lambda-1-(\alpha+1) / p} K(1, t) d t<\infty
$$

Moreover, when (2.11) holds, the operator norm $\|T\|$ of $T$ on $L^{p}\left(\omega_{2}\right)$ satisfies

$$
\|T\|=\int_{0}^{\infty} t^{\lambda-1-(\alpha+1) / p} K(1, t) d t .
$$

If $K(x, y)=\frac{1}{x} \varphi_{(0, x)}(y), \lambda>\frac{\alpha+1}{p}$, then by (1.3) and (2.12), we reduced the classical Hardy operator

$$
T_{0}(f, x)=\frac{1}{x} \int_{0}^{x} f(y) d y
$$

and

$$
\left\{\int_{0}^{\infty}\left|\frac{1}{x} \int_{0}^{x} f(y) d y\right|^{p} x^{\alpha} d x\right\}^{1 / p} \leq \frac{p}{\lambda p-\alpha-1}\left(\int_{0}^{\infty}|f(x)|^{p} x^{(1-\lambda) p+\alpha} d x\right)^{1 / p} .
$$

where $\left\|T_{0}\right\|=p /(\lambda p-\alpha-1)$ is the best possible constant. In pacticular, when $\alpha=0$, $\lambda=1$, (2.13) reduce to the classical Hardy inequality $[3,4]$ :

$$
\left(\int_{0}^{\infty}\left|\frac{1}{x} \int_{0}^{x} f(t) d t\right|^{p} d x\right)^{1 / p} \leq \frac{p}{p-1}\left(\int_{0}^{\infty}|f(x)|^{p} d x\right)^{1 / p}
$$


Hence, our main results imply many useful inequalities with the best constant factors.

There are some similar results for the non-homogeneous Herz spaces. We omit the details here.

\section{Proofs of Theorems 1 and 3}

In what follows, we shall write simply $\dot{K}_{q}^{\alpha, p}\left(R_{+}^{1}\right)$ and $\dot{K}_{q}^{\alpha, p}(\omega)$ to denote $K$ and $K(\omega)$, respectively.

Proof of Theorem 1. Using Minkowski inequality for integrals and setting $y=t x$, we get

$$
\begin{aligned}
\left\|(T f) \varphi_{k}\right\|_{q} & =\left\{\int_{D_{k}}\left|\int_{0}^{\infty} K(x, y) f(y) d y\right|^{q} d x\right\}^{1 / q} \\
& \leq \int_{0}^{\infty}\left(\int_{D_{k}}|f(t x)|^{q} x^{(1-\lambda) q} d x\right)^{1 / q} K(1, t) d t \\
& =\int_{0}^{\infty}\left(\int_{2^{k-1} t<y \leq 2^{k} t}|f(y)|^{q} y^{(1-\lambda) q} d y\right)^{1 / q} t^{\lambda-1-1 / q} K(1, t) d t .
\end{aligned}
$$

For each $t \in(0, \infty)$, there exists an integer $m$ such that $2^{m-1}<t \leq 2^{m}$. Setting

$$
A_{k, m}=\left\{y \in(0, \infty): 2^{k+m-1}<y \leq 2^{k+m}\right\}
$$

we obtain

$$
\begin{aligned}
\left\|(T f) \varphi_{k}\right\|_{q} & \leq \int_{0}^{\infty}\left(\int_{A_{(k-1), m}}|f(y)|^{q} \omega(y) d y+\int_{A_{k, m}}|f(y)|^{q} \omega(y) d y\right)^{1 / q} t^{\lambda-1-1 / q} K(1, t) d t \\
& \leq \int_{0}^{\infty}\left(\left\|f \varphi_{k+m-1}\right\|_{q, \omega}+\left\|f \varphi_{k+m}\right\|_{q, \omega}\right) t^{\lambda-1-1 / q} K(1, t) d t .
\end{aligned}
$$

It follows that

$$
\|T f\|_{K} \leq\left\{\sum_{k=-\infty}^{\infty} 2^{k \alpha p}\left[\int_{0}^{\infty}\left(\left\|f \varphi_{k+m-1}\right\|_{q, \omega}+\left\|f \varphi_{k+m}\right\|_{q, \omega}\right) t^{\lambda-1-1 / q} K(1, t) d t\right]^{p}\right\}^{1 / p} .
$$

Now, we consider two cases for $p$ :

Case 1. $0<p<1$. In this case, it follows from (3.1), (2.6) and (2.5) that

$$
\|T f\|_{K} \leq C_{2}(p)\left\{\sum_{k=-\infty}^{\infty} 2^{k \alpha p} \int_{0}^{\infty}\left(\left\|f \varphi_{k+m-1}\right\|_{q, \omega}^{p}+\left\|f \varphi_{k+m}\right\|_{q, \omega}^{p}\right)\right.
$$




$$
\begin{aligned}
& \left.\times t^{(\lambda-1-1 / q) p} K^{p}(1, t) d t\right\}^{1 / p} \\
\leq & 2^{1 / p-1} C_{2}(p)\left\{\left[\sum_{k=-\infty}^{\infty} 2^{(k+m-1) \alpha p}\left\|f \varphi_{k+m-1}\right\|_{q, \omega}^{p}\right.\right. \\
& \left.\times \int_{0}^{\infty} 2^{-(m-1) \alpha p} t^{(\lambda-1-1 / q) p} K^{p}(1, t) d t\right]^{1 / p} \\
& \left.+\left[\sum_{k=-\infty}^{\infty} 2^{(k+m) \alpha p}\left\|f \varphi_{k+m}\right\|_{q, \omega}^{p} \int_{0}^{\infty} 2^{-m \alpha p} t^{(\lambda-1-1 / q) p} K^{p}(1, t) d t\right]^{1 / p}\right\} \\
\leq & 2^{1 / p-1}\left\{C_{1}(1 / p)\right\}^{1 / p} C_{2}(p)\|f\|_{K(\omega)} \int_{0}^{\infty}\left(2^{-(m-1) \alpha}+2^{-m \alpha}\right) t^{\lambda-1-1 / q} K(1, t) d t \\
\leq & 2^{1 / p-1}\left\{C_{1}(1 / p)\right\}^{1 / p} C_{2}(p)\left(1+2^{|\alpha|}\right)\|f\|_{K(\omega)} \int_{0}^{\infty} t^{\lambda-\alpha-1-1 / q} K(1, t) d t . \quad(3.2)
\end{aligned}
$$

Case 2. $1 \leq p<\infty$. In this case, it follows from (3.1), (2.5) and (2.6) that

$$
\begin{aligned}
\|T f\|_{K} \leq & C_{1}(p)\left\{\sum_{k=-\infty}^{\infty} 2^{k \alpha p} \int_{0}^{\infty}\left(\left\|f \varphi_{k+m-1}\right\|_{q, \omega}+\left\|f \varphi_{k+m}\right\|_{q, \omega}\right)^{p}\right. \\
& \left.\times t^{(\lambda-1-1 / q) p} K^{p}(1, t) d t\right\}^{1 / p} \\
\leq & 2^{1-1 / p} C_{1}(p)\left\{\left[\sum_{k=-\infty}^{\infty} 2^{(k+m-1) \alpha p}\left\|f \varphi_{k+m-1}\right\|_{q, \omega}^{p}\right.\right. \\
& \left.\times \int_{0}^{\infty} 2^{-(m-1) \alpha p} t^{(\lambda-1-1 / q) p} K^{p}(1, t) d t\right]^{1 / p} \\
& \left.+\left[\sum_{k=-\infty}^{\infty} 2^{(k+m) \alpha p}\left\|f \varphi_{k+m}\right\|_{q, \omega}^{p} \int_{0}^{\infty} 2^{-m \alpha p} t^{(\lambda-1-1 / q) p} K^{p}(1, t) d t\right]^{1 / p}\right\} \\
\leq & 2^{1-1 / p} C_{1}(p) C_{2}(1 / p)^{1 / p}\|f\|_{K(\omega)} \int_{0}^{\infty}\left(2^{-(m-1) \alpha}+2^{-m \alpha}\right) t^{\lambda-1-1 / q} K(1, t) d t \\
\leq & 2^{1-1 / p} C_{1}(p) C_{2}(1 / p)^{1 / p}\left(1+2^{|\alpha|}\right)\|f\|_{K(\omega)} \int_{0}^{\infty} t^{\lambda-\alpha-1-1 / q} K(1, t) d t .
\end{aligned}
$$

Hence, by (3.2) and (3.3), we get

$$
\|T\| \leq C(p, \alpha) \int_{0}^{\infty} t^{\lambda-\alpha-1-1 / q} K(1, t) d t .
$$

where $C(p, \alpha)$ is defined by $(2.9)$. 
To prove the opposite inequality, we set for each $\varepsilon \in(0,1)$,

$$
f_{\varepsilon}(x)=\left\{\begin{array}{cc}
0, & 0<x \leq 1, \\
x^{\lambda-\alpha-1-1 / q-\varepsilon}, & x>1
\end{array}\right.
$$

then

$$
\left\|f_{\varepsilon} \varphi_{k}\right\|_{q, \omega}^{q}=\int_{2^{k-1}<x \leq 2^{k}} x^{(\lambda-\alpha-1-1 / q-\varepsilon) q} x^{(1-\lambda) q} d x=C_{3} 2^{-k(\alpha+\varepsilon) q}
$$

where

$$
C_{3}=\left|\frac{2^{(\alpha+\varepsilon) q}-1}{(\alpha+\varepsilon) q}\right|
$$

It follows that

$$
\left\|f_{\varepsilon}\right\|_{K(\omega)}=\left\{\sum_{k=-\infty}^{\infty} 2^{k \alpha p}\left(C_{3}^{1 / q} 2^{-k(\alpha+\varepsilon)}\right)^{p}\right\}^{1 / p}=C_{3}^{1 / q} \frac{2^{-\varepsilon}}{\left(1-2^{-\varepsilon p}\right)^{1 / p}} .
$$

Observe that

$$
T\left(f_{\varepsilon}, x\right)=x^{-(\alpha+1 / q+\varepsilon)} \int_{x^{-1}}^{\infty} t^{\lambda-(\alpha+1+1 / q+\varepsilon)} K(1, t) d t .
$$

For each $\varepsilon \in(0,1)$, there exists a positive integer $l$, such that $2^{l-1} \leq 1 / \varepsilon<2^{l}$, so that

$$
\begin{aligned}
\left\|T f_{\varepsilon}\right\|_{K}^{p} & =\sum_{k=1}^{\infty} 2^{k \alpha p}\left\{\int_{x>1}\left[T\left(f_{\varepsilon}, x\right) \varphi_{k}(x)\right]^{q} d x\right\}^{p / q} \\
& =\sum_{k=1}^{\infty} 2^{k \alpha p}\left\{\int_{2^{k-1}<x \leq 2^{k}} x^{-(\alpha+1 / q+\varepsilon) q}\left(\int_{x^{-1}}^{\infty} t^{\lambda-(\alpha+1+1 / q+\varepsilon)} K(1, t) d t\right)^{q} d x\right\}^{p / q} \\
& \geq \sum_{k=l+1}^{\infty} 2^{k \alpha p}\left(\int_{\varepsilon}^{\infty} t^{\lambda-(\alpha+1+1 / q+\varepsilon)} K(1, t) d t\right)^{p}\left(\int_{2^{k-1}<x \leq 2^{k}} x^{-(\alpha+1 / q+\varepsilon) q} d x\right)^{p / q} \\
& =\left(\int_{\varepsilon}^{\infty} t^{\lambda-(\alpha+1+1 / q+\varepsilon)} K(1, t) d t\right)^{p} \sum_{k=l+1}^{\infty} 2^{k \alpha p}\left(C_{3} 2^{-k(\alpha+\varepsilon) q}\right)^{p / q} \\
& =C_{3}^{p / q}\left(\int_{\varepsilon}^{\infty} t^{\lambda-(\alpha+1+1 / q+\varepsilon)} K(1, t) d t\right)^{p} \frac{2^{-\varepsilon p(l+1)}}{1-2^{-\varepsilon p}} .
\end{aligned}
$$

Thus,

$$
\|T\| \geq \frac{\left\|T f_{\varepsilon}\right\|_{K}}{\left\|f_{\varepsilon}\right\|_{K(\omega)}} \geq 2^{-\varepsilon l} \int_{\varepsilon}^{\infty} t^{\lambda-(\alpha+1+1 / q+\varepsilon)} K(1, t) d t .
$$

Take limits as $\varepsilon \rightarrow 0$ in (3.9), we obtain

$$
\|T\| \geq \int_{0}^{\infty} t^{\lambda-(\alpha+1+1 / q)} K(1, t) d t .
$$

This finishes the proof of Theorem 1. 
Proof of Theorem 3. By Minkowsks inequality for integrals and setting $y=t x$, we have

$$
\begin{aligned}
\|T f\|_{p, \omega_{1}} & \leq\left\{\int_{0}^{\infty}\left(\int_{0}^{\infty} K(x, y)|f(y)| d y\right)^{p} x^{\alpha} d x\right\}^{1 / p} \\
& \leq \int_{0}^{\infty}\left\{\int_{0}^{\infty}|f(t x)|^{p} x^{(1-\lambda) p+\alpha} d x\right\}^{1 / p} K(1, t) d t \\
& =\|f\|_{p, \omega_{2}} \int_{0}^{\infty} t^{\lambda-1-(\alpha+1) / p} K(1, t) d t .
\end{aligned}
$$

It follows that

$$
\|T\|=\sup _{f \neq 0} \frac{\|T f\|_{p, \omega_{1}}}{\|f\|_{p, \omega_{2}}} \leq \int_{0}^{\infty} t^{\lambda-1-(\alpha+1) / p} K(1, t) d t .
$$

To prove the opposite inequality, we set for any $\varepsilon \in(0,1)$,

$$
f_{\varepsilon}(x)=\left\{\begin{array}{cc}
0, & 0<x \leq 1 \\
x^{\lambda-1-(1+\alpha) / p-\varepsilon}, & x>1
\end{array}\right.
$$

then

$$
\left\|f_{\varepsilon}\right\|_{p, \omega_{2}}^{p}=\int_{1}^{\infty} x^{-p \varepsilon-1} d x=\frac{1}{p \varepsilon}
$$

Thus,

$$
\begin{aligned}
\left\|T f_{\varepsilon}\right\|_{p, \omega_{1}} & =\left(\int_{x>1}\left|T\left(f_{\varepsilon}, x\right)\right|^{p} x^{\alpha} d x\right)^{1 / p} \\
& =\left\{\int_{x>1} x^{-(1+p \varepsilon)}\left(\int_{x^{-1}}^{\infty} t^{\lambda-1-(1+\alpha) / p-\varepsilon} K(1, t) d t\right)^{p} d x\right\}^{1 / p} \\
& \geq\left(\int_{\varepsilon}^{\infty} t^{\lambda-1-(1+\alpha) / p-\varepsilon} K(1, t) d t\right)(p \varepsilon)^{-1 / p}
\end{aligned}
$$

This implies

$$
\|T\| \geq \frac{\left\|T f_{\varepsilon}\right\|_{p, \omega_{1}}}{\left\|f_{\varepsilon}\right\|_{p, \omega_{2}}} \geq \int_{\varepsilon}^{\infty} t^{\lambda-1-(1+\alpha) / p-\varepsilon} K(1, t) d t .
$$

Take limits as $\varepsilon \rightarrow 0$ in (3.12), we get

$$
\|T\| \geq \int_{0}^{\infty} t^{\lambda-1-(1+\alpha) / p} K(1, t) d t .
$$

Then by (3.12) and (3.13), we have $\|T\|=\int_{0}^{\infty} t^{\lambda-1-(1+\alpha) / p} K(1, t) d t$. The Theorem is proved. 


\section{References}

[1] G. H. Hardy, J. E. Littlewood and G. Polya, Inequalities, $2^{\text {nd }}$ ed. Cambridge University Press, London, 1952.

[2] E. F. Beckenbach and R. Bellman, Inequalities, Springer-Verlag, Berlin, 1983.

[3] Kuang Jichang, Applied Inequalities, $3^{\text {rd }}$ Ed. Shangdong Science Press, Jinan, 2004.

[4] Alois Kufner, Lech Maligranda and Lars-Erik Persson, The Hardy inequality, Pilsen, 2007.

[5] Bicheng Yang and T. M. Rassias, On the way of weight coefficient and research for the Hilbert-type inequalities, Math. Ineq. \& Appl. 6(2003), 625-658.

[6] Gao Ming-zhe and L. C. Hsu, A survey of various refinements and generalizations of Hilbert's inequalities, J. Math. Res. Exposition 25(2005), 227-243.

[7] Kuang Jichang, On new extensions of Hilbert's integral inequality, J. Math. Anal. Appl. 235(1999), 608-614.

[8] Kuang Jichang and T. M. Rassias, Hilbert integral operator inequalities, Math. Ineq. \& Appl. 3(2000), 497-510.

[9] Kuang Jichang and L. Debnath, On new generalization of Hilbert's inequality and their applications, J. Math. Anal. Appl. 245(2000), 248-265.

[10] Kuang Jichang and L. Debnath, The general form of Hilbert's inequality and its converses, Analysis Math. 31(2005), 163-173.

[11] Kuang Jichang and L. Debnath, On Hilbert's type inequalities on the weighted Orlicz spaces, Pacific J. of Appl. Math. 1(2007), 95-103.

[12] S. Lu and D. Yang, The weighted Herz-type Hardy spaces and its applications, Sci. in China, (Ser.A), 38(1995), 662-673.

[13] Eugenio Hernandez, Guido Weiss, and Dachun Yang, The $\varphi$-transform and wavelet charac-terizations of Herz-type spaces, Collect. Math. 47(1996), 285-320.

Department of Mathematics, Hunan Normal University, Changsha, Hunan, 410081, P.R.CHINA

E-mail: jc-kuang@hotmail.com 